\title{
Diagnostic Accuracy of Lymph Node Metastasis Depends on Metabolic Activity of the Primary Lesion in Thoracic Squamous Esophageal Cancer
}

\author{
Osamu Manabe ${ }^{1}$, Naoya Hattori ${ }^{1}$, Kenji Hirata $^{1}$, Kazuo Itoh ${ }^{2}$, Masao Hosokawa ${ }^{3}$, Hiroaki Takahashi ${ }^{4}$, \\ Noriko Oyama-Manabe ${ }^{5}$, and Nagara Tamaki ${ }^{1}$ \\ ${ }^{I}$ Department of Nuclear Medicine, Hokkaido University Graduate School of Medicine, Sapporo, Japan; ${ }^{2}$ Department of Radiology, \\ Keiyukai Sapporo Hospital, Sapporo, Japan; ${ }^{3}$ Department of Surgery, Keiyukai Sapporo Hospital, Sapporo, Japan; ${ }^{4}$ Department of \\ Internal Medicine, Keiyukai Sapporo Hospital, Sapporo, Japan; and ${ }^{5}$ Department of Diagnostic and Interventional Radiology, \\ Hokkaido University Hospital, Sapporo, Japan
}

The metabolic activity of the primary tumor is an important variable in ${ }^{18} \mathrm{~F}-\mathrm{FDG}$ PET interpretation for presurgical staging, because this activity is likely to affect the possibility of detection of malignant involvement in lymph nodes (LNs). The purpose of this study was to reevaluate the diagnostic accuracy of ${ }^{18} \mathrm{~F}$ FDG PET/CT for the presurgical staging of esophageal squamous cell carcinoma (SCC) in correlation with the ${ }^{18} \mathrm{~F}-\mathrm{FDG}$ avidity of the primary lesions. Methods: One hundred fifty-six patients (mean age $\pm \mathrm{SD}, 61.4 \pm 8.0 \mathrm{y}$ ) underwent ${ }^{18} \mathrm{~F}-\mathrm{FDG}$ $\mathrm{PET} / \mathrm{CT}$ before surgical esophagectomy and LN dissection. LN metastasis was identified using the fusion of PET and CT images with increased ${ }^{18} \mathrm{~F}-\mathrm{FDG}$ uptake greater than the background activity of the adjacent structures. The results of the patients' ${ }^{18} \mathrm{~F}-\mathrm{FDG}$ PET/CT examinations for LN involvement were compared with the histopathologic results to investigate the diagnostic accuracy of ${ }^{18} \mathrm{~F}-\mathrm{FDG}$ PET/CT for tumor staging. In addition, we examined the correlation between the diagnostic accuracy of ${ }^{18} \mathrm{~F}-\mathrm{FDG}$ PET/CT for LN involvement and the ${ }^{18} \mathrm{~F}$ FDG avidity of the primary lesions, to investigate the effect of tumor aggressiveness on the diagnosis of LN metastasis. Results: The diagnostic accuracy of ${ }^{18}$ F-FDG PET/CT for LN metastasis showed a low sensitivity, ranging from $29.3 \%$ to $53.3 \%$, whereas the specificity was higher than $89.8 \%$ in regional thoracic nodes and in remote areas of the cervical and abdominal regions. The ${ }^{18} \mathrm{~F}-\mathrm{FDG}$ uptake of the primary lesions positively correlated with that of the metastatic LNs in the thoracic field $(R=0.52, P<0.05)$. As a result, our receiver-operatingcharacteristic analyses demonstrated an area under the curve value of 0.73 , with the optimal cutoff value at a maximum standardized uptake value of 3.3 in patients with mid to high ${ }^{18} \mathrm{~F}$ FDG avidity in the primary lesions (maximum standardized uptake value $\geq 5$ ). Conclusion: This study showed that the avidity of the primary esophageal SCCs affected the detectability of lymph nodal metastases. If primary lesions of esophageal SCC present with a low ${ }^{18} \mathrm{~F}-\mathrm{FDG}$ uptake, PET/CT may have

\footnotetext{
Received Jun. 21, 2012; revision accepted Nov. 26, 2012.

For correspondence or reprints contact: Naoya Hattori, N15 W7, Kita-Ku, Sapporo, 0608638 Hokkaido, Japan.

E-mail: nhattori@med.hokudai.ac.jp

Published online Mar. 20, 2013.

COPYRIGHT (C 2013 by the Society of Nuclear Medicine and Molecular Imaging, Inc.
}

a limited role for initial staging because of low sensitivity to detect lymph node metastases.

Key Words: esophageal cancer; squamous cell carcinoma; FDG PET; pathology

J Nucl Med 2013; 54:670-676

DOI: 10.2967/jnumed.112.110304

$\mathbf{E}$ nancy of the digestive tract and a leading cause of cancer mortality worldwide, with an estimated 5-y survival of $15 \%$ (1). Even after potentially curative surgery, long-term survival rates rarely exceed $35 \%$ (2). The available therapeutic options for esophageal cancer vary depending on the tumor stage, and therefore accurate pretherapeutic staging is crucial to the selection of the appropriate type of therapy (3).

There have been multiple investigations of the clinical utility of ${ }^{18}$ F-FDG PET for the presurgical staging of esophageal cancer, and the investigators generally concluded that ${ }^{18}$ F-FDG PET has a limited role in the identification of early regional lymph node (LN) metastasis but is highly useful for detecting remote lymph nodal involvement and metastasis to remote organs $(4,5)$. A nonspecific inflammatory process probably explains the poor detection of regional LN metastasis. However, the aggressiveness of the primary tumor may also influence the metabolic activity of the LNs, which may affect the ability of ${ }^{18} \mathrm{~F}-\mathrm{FDG}$ PET/CT to accurately demonstrate LN metastasis. In non-small cell lung cancer (NSCLC), ${ }^{18} \mathrm{~F}$-FDG uptake within the primary lesion correlates with aggressiveness on PET studies, indicating intratumoral lymphatic vessel invasion and LN involvement $(6,7)$. The metabolic activity of the primary tumor and tumor size are important variables in ${ }^{18} \mathrm{~F}-\mathrm{FDG}$ PET interpretation for NSCLC, because they affect the likelihood of malignant involvement in nodes $(8)$.

For esophageal cancer with squamous cell carcinoma (SCC), there have been no established findings regarding 
the ${ }^{18} \mathrm{~F}-\mathrm{FDG}$ uptake of the primary lesions and LN metastasis. The diagnostic accuracy of ${ }^{18} \mathrm{~F}$-FDG PET/CT for the presurgical staging of esophageal cancer should be reevaluated on the basis of the ${ }^{18}$ F-FDG avidity of the primary lesions. The purpose of the present study was to evaluate the clinical utility of ${ }^{18} \mathrm{~F}$-FDG PET/CT for the presurgical staging of thoracic esophageal cancer with SCC pathology. We investigated the diagnostic ability of ${ }^{18} \mathrm{~F}-\mathrm{FDG}$ PET/CT to accurately detect metastasized LNs, and we examined this diagnostic accuracy in relation to the metabolic activity of the primary lesions. We attempted to evaluate the accuracy of this imaging modality for identifying lymph nodal metastasis in regional (thoracic) versus remote (cervical and abdominal) fields separately, to clarify the possible regional differences in the diagnostic value of ${ }^{18} \mathrm{~F}-\mathrm{FDG}$ PET/ CT. We also investigated the possibility of distinguishing true- versus false-positive LN metastasis, using a semiquantitative value of the maximum standardized uptake value (SUVmax). This was a retrospective study, conducted in a clinical center that specializes in the surgical treatment of esophageal cancer.

\section{MATERIALS AND METHODS}

\section{Study Design}

Patients with known esophageal cancer underwent ${ }^{18} \mathrm{~F}$-FDG PET/CT before esophagectomy. Their PET/CT images were used for the diagnosis of esophageal cancer and for presurgical clinical staging based on the TNM classification of the American Joint Committee on Cancer, seventh edition (9). LN involvement was visually and quantitatively inspected in the cervical, thoracic, and abdominal regions. The results of each patient's ${ }^{18} \mathrm{~F}$-FDG PET/CT examination for $\mathrm{LN}$ involvement were compared with their histopathology results to investigate the diagnostic accuracy of ${ }^{18} \mathrm{~F}-\mathrm{FDG}$ PET/CT for tumor stating. In particular, the diagnostic accuracy of $\mathrm{LN}$ involvement was correlated with the ${ }^{18} \mathrm{~F}-\mathrm{FDG}$ avidity of the primary lesion to investigate the effect of tumor aggressiveness on the diagnosis of $\mathrm{LN}$ metastasis. This is a retrospective study on a population that underwent a standard treatment procedure at the institution. All patients gave written informed consent before $\mathrm{PET} / \mathrm{CT}$ for the possible future use of their anonymized clinical data for scientific or clinical research purpose. The institutional review board at Keiyukai Sapporo Hospital, Sapporo, Japan, approved the retrospective analyses and the publication of the study results.

TABLE 1

Sensitivity of Primary Lesions

\begin{tabular}{lcrc} 
PT stage & ${ }^{18}$ F-FDG positive & Total & Sensitivity (\%) \\
\hline T1a & 10 & 16 & 62.5 \\
T1b & 42 & 57 & 73.7 \\
T2 & 12 & 12 & 100 \\
T3 & 69 & 69 & 100 \\
T4 & 2 & 2 & 100 \\
Total & 135 & 156 & 86.5 \\
\hline
\end{tabular}

\section{Subjects}

The study population retrospectively included patients with esophageal cancer who underwent ${ }^{18}$ F-FDG PET/CT before esophagectomy from April 2006 to June 2008. The inclusion criteria were a primary lesion located in the thoracic esophagus, histologically proven SCC, and PET/CT performed before surgical esophagectomy and LN dissection. Patients who met the following criteria were excluded from the study: coexisting cancer that was located outside the thoracic esophagus or the primary lesion had been treated with chemotherapy or radiotherapy before PET/CT acquisition. The final study population comprised 156 patients (125 men and 31 women), ranging in age from 40 to 84 y (mean age \pm SD, $61.4 \pm 8.0$ y). Serum glucose level was $100.4 \pm 13.1 \mathrm{mg} / \mathrm{dL}$ (range, $67-171 \mathrm{mg} / \mathrm{dL}$ ) before ${ }^{18} \mathrm{~F}-\mathrm{FDG}$ administration.

\section{Pathologic Assessment}

All patients underwent surgical $\mathrm{LN}$ dissection at the same time as the esophagectomy. Specimens from the primary tumors were histopathologically classified based on T factors. T1 stage tumors were classified as T1a when the tumor invaded into but not through the muscularis mucosa and as $\mathrm{T} 1 \mathrm{~b}$ when there was submucosal invasion. For the LN involvement, 3-field (cervical, thoracic, and abdominal) LN dissections were performed in 147 patients, and 2-field (thoracic and abdominal) dissections were performed in the remaining 9 patients. All of the LNs in the 3 surgical fields were dissected, and their locations were identified according to the TNM classification system of the American Joint Committee on Cancer. A board-certified pathologist performed the standard histopathologic examination with hematoxylin and eosin staining for both primary lesions and dissected LNs. The LN involvement was summarized in regional (thoracic) and remote (cervical and abdominal) categories to determine the postsurgical therapeutic strategy as proposed in the previous literature (10).

\section{PET/CT Imaging}

All patients underwent PET/CT scanning with a GEMINI GXL (Philips Healthcare). The matrix size was $144 \times 144$, and the full width at half maximum was $5.6 \mathrm{~mm}$ (11), with multidetector CT. The imaging protocol required that the patient fast for at least $4 \mathrm{~h}$ before the injection of ${ }^{18}$ F-FDG. A mean dose of $241.3 \pm$ $70.0 \mathrm{MBq}$ of ${ }^{18} \mathrm{~F}-\mathrm{FDG}$ was administered intravenously, which was $4.23 \pm 0.98 \mathrm{MBq}$ per $\mathrm{kg}$ of body weight. After the injection, the patient rested comfortably on a sofa for $60 \mathrm{~min}$. Image acquisition was initiated with a CT scan for attenuation correction and anatomic localization (30-80 mA, $120 \mathrm{keV}$ ), followed by 3dimensional PET acquisition with 2 min per bed position, covering from the vertex to the upper thighs. Acquired datasets were corrected for attenuation, dead-time, and scatter, and the images were reconstructed using the line-of-response row-action maximumlikelihood algorithm method (12).

\section{Image Analyses}

Two nuclear medicine physicians visually interpreted the reconstructed images to identify the primary lesions and LN metastases, using a 3-dimensional computer display workstation in consensus. The locations of the primary lesions were sought using the endoscopic examination report as a reference, because earlystage esophageal cancers often infiltrate the mucosal surface of the esophagus, thus limiting the visualization on a CT image. LN metastasis was identified using the fusion of PET and CT images with increased ${ }^{18}$ F-FDG uptake greater than the background activity of the blood pool. The symmetric hilum accumulations were 


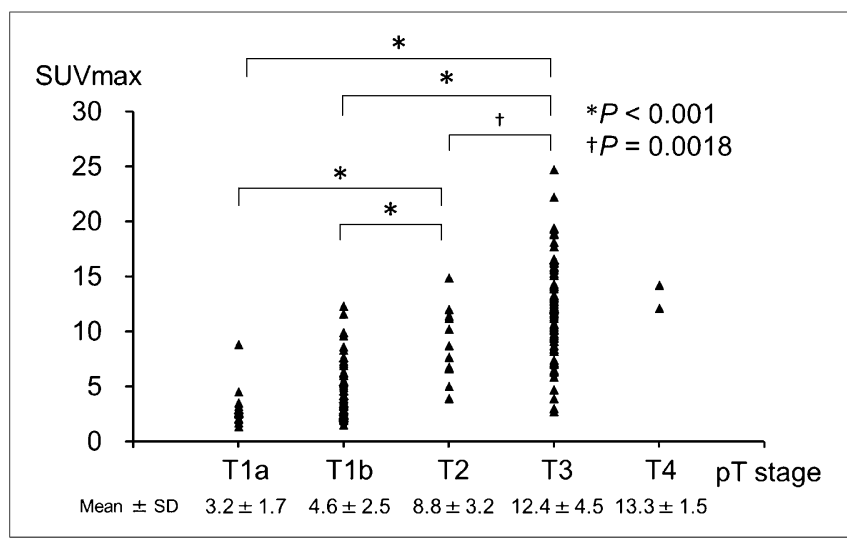

FIGURE 1. SUVmax of primary lesions among different pT stages.

excluded from the analysis, because esophageal cancer rarely metastasizes to the hilum (13), and the symmetric hilar uptake generally represents a chronic inflammatory process. For the quantitative analysis, regions of interest were manually placed on primary lesions and nodal metastases, to obtain SUVmax.

\section{Statistical Analysis}

Data are expressed as mean \pm SD. The correlation between the SUVmax of the primary lesions and $\mathrm{T}$ stage was analyzed using a Spearman rank correlation because $\mathrm{T}$ stage was discrete. The correlations of SUVmax between primary esophageal cancer and LN metastases were analyzed using Pearson correlation coefficients. For both analyses, $P$ values less than 0.05 were considered significant. The diagnostic accuracy of ${ }^{18} \mathrm{~F}-\mathrm{FDG}$ PET/CT for LN metastases was investigated using receiver-operating-characteristics (ROC) analyses.

\section{RESULTS}

\section{Primary Tumors}

Table 1 summarizes the sensitivity of the primary lesions. Visual inspection of the ${ }^{18} \mathrm{~F}-\mathrm{FDG}$ PET/CT images failed to identify the primary lesion for 21 of the 73 patients with T1 tumors (6/16 in T1a and 15/57 in T1b), but it succeeded in detecting all of the primary tumors more advanced than $\mathrm{T} 1$ stage. The mean SUVmax was positively correlated with the T stage of the tumors $(P<0.0001)$, although there were significant overlaps among the different $\mathrm{T}$ stages (Fig. 1). The distribution of SUVmax of the primary lesions was determined for all 156 patients: 58 presented with low ${ }^{18} \mathrm{~F}-\mathrm{FDG}$
TABLE 2

${ }^{18}$ F-FDG PET/CT and Pathologic Findings of LN Metastasis

\begin{tabular}{lrc}
\hline N stage & PET & Pathology \\
\hline N0 & 100 & 67 \\
N1 & 50 & 46 \\
N2 & 6 & 23 \\
N3 & 0 & 20 \\
\hline
\end{tabular}

avidity (SUVmax $<5$ ), 41 with medium ${ }^{18}$ F-FDG avidity $(5 \leq$ SUVmax $<10)$, and 57 with high ${ }^{18}$ F-FDG avidity $($ SUVmax $\geq 10)$.

\section{LN Metastases}

Compared with the histopathologic analyses, ${ }^{18} \mathrm{~F}-\mathrm{FDG}$ $\mathrm{PET} / \mathrm{CT}$ tended to underestimate $\mathrm{N}$ staging. For the $\mathrm{N}$ staging, PET/CT agreed with the histopathologic findings in 85 of $156(54.5 \%)$ patients, underestimated stage in 64 of 156 $(41.0 \%)$, and overestimated stage in 7 of $156(4.5 \%)$. Among the 156 patients investigated, ${ }^{18} \mathrm{~F}-\mathrm{FDG}$ PET/CT showed visually abnormal ${ }^{18} \mathrm{~F}$-FDG uptake in $56(35.9 \%)$ patients in 1 of the regional LNs ( $\geq \mathrm{N} 1)$, indicating possible metastasis, whereas the histopathologic analysis demonstrated regional micrometastasis in $89(57.1 \%)$ patients (Table 2).

\section{${ }^{18}$ F-FDG Avidity of Primary Lesion and LN Metastasis}

${ }^{18} \mathrm{~F}-\mathrm{FDG}$ avidity of the primary lesion was positively associated with the prevalence of pathologically confirmed LN micrometastasis (Table 3). As a result, positive predictive values (PPVs) of visual inspection of ${ }^{18} \mathrm{~F}-\mathrm{FDG}$ PET/CT were lower in patients with low ${ }^{18} \mathrm{~F}-\mathrm{FDG}$ avidity in the primary lesions (SUVmax $<5$ ) than in patients with medium and high ${ }^{18}$ F-FDG avidity (SUVmax $\geq 5$ ) in the primary lesions (Table 4).

\section{Association of ${ }^{18}$ F-FDG Avidity Between Primary and LN Metastases}

The ${ }^{18}$ F-FDG avidity of the primary lesion also affected metabolic activity of metastatic LNs. Higher ${ }^{18}$ F-FDG uptake in the primary lesion generally associated with pathologic LN metastasis, which was also positive with visual inspection of PET/CT (Fig. 2). In particular, ${ }^{18} \mathrm{~F}-\mathrm{FDG}$ uptake

TABLE 3

Pathologic N Stages Among Each Avidity Group of Main Lesion

\begin{tabular}{lccr}
\hline & & \multicolumn{2}{c}{ Avidity } \\
\cline { 2 - 3 } N stage & Low $($ SUVmax $<5)$ & Mid $(5 \leq$ SUVmax $<10)$ \\
N0 & $35(60.3)$ & $15(36.6)$ & $17(29.8)$ \\
N1 & $15(25.9)$ & $10(24.4)$ & $21(36.8)$ \\
N2 & $6(10.3)$ & $9(22.0)$ & $7(17.1)$ \\
N3 & $2(3.4)$ & 41 & $11(19.0)$ \\
Total & 58 & 57
\end{tabular}

Data in parentheses are percentages. 
TABLE 4

Diagnostic Value of Nodal Involvements by Activity of Main Lesion

\begin{tabular}{|c|c|c|c|c|c|c|}
\hline \multirow[b]{2}{*}{ Parameter } & \multicolumn{2}{|c|}{ All nodes } & \multicolumn{2}{|c|}{ Regional nodes } & \multicolumn{2}{|c|}{ Remote nodes } \\
\hline & Low & Mid to high & Low & Mid to high & Low & Mid to high \\
\hline Sensitivity (\%) & 15.2 & 41.5 & 15.4 & 33.7 & 15.0 & 46.9 \\
\hline Specificity (\%) & 95.7 & 92.3 & 91.1 & 89.6 & 97.9 & 93.5 \\
\hline Positive predictive value (\%) & 45.5 & 76.3 & 33.3 & 74.0 & 60.0 & 78.3 \\
\hline Negative predictive value (\%) & 82.8 & 71.3 & 78.8 & 61.6 & 84.7 & 76.4 \\
\hline
\end{tabular}

of pathologically confirmed LN metastases positively correlated with that of the primary lesions $(R=0.52, P<$ 0.05 ; Fig. 3 ) in the thoracic region, although the association did not reach the level of significance in the remote regions. The metabolic activity of the primary lesion affected the sensitivity of visual inspection of ${ }^{18}$ F-FDG PET/CT for LN metastasis (Fig. 4).

\section{Best-Cutoff Criteria for LN Metastasis}

In accordance with the visual inspection, ${ }^{18} \mathrm{~F}-\mathrm{FDG}$ avidity of the primary lesion affected the semiquantitative approach of diagnosing LN metastases (Figs. 5A-5C). Discrimination between metastatic versus reactive LNs was difficult using SUVmax in patients with low ${ }^{18} \mathrm{~F}$-FDG avidity in the primary lesions (SUVmax $<5$ ), with an area under the curve of 0.56 (Fig. 5B). In contrast, this semiquantitative approach was fairly successful at diagnosing LN metastases in patients with medium to high ${ }^{18} \mathrm{~F}-\mathrm{FDG}$ avidity in the primary lesions (SUVmax $\geq 5$ ), with an area under the curve of 0.73. The ROC analysis revealed the best discrimination of true- versus false-positives (highest sensitivity - (1 - specificity)) at the SUVmax of 3.3 (Fig. 5C). The application of this cutoff value (SUVmax, 3.3) resulted in the sensitivity and specificity of $66.1 \%$ and $85.7 \%$, respectively, to identify metastatic LNs with visually positive ${ }^{18} \mathrm{~F}-\mathrm{FDG}$ uptake. Use of the same cutoff value on the patients with mid to high ${ }^{18} \mathrm{~F}$ FDG uptake in the primary lesion improved the PPV from $74.0 \%$ (visual) to $87.5 \%$ (semiquantitative) in regional (thoracic) LNs. The same cutoff value resulted in $100 \%$ PPV for remote-region metastasis (Table 5).

\section{DISCUSSION}

The visual inspection of ${ }^{18} \mathrm{~F}-\mathrm{FDG}$ PET/CT images had limited sensitivity to detect LN metastasis, ranging from $29.3 \%$ to $53.3 \%$ in regional thoracic nodes and in remote areas of the cervical and abdominal regions. However, ${ }^{18} \mathrm{~F}-$ FDG PET/CT of aggressive esophageal cancers had a better diagnostic accuracy, because the ${ }^{18} \mathrm{~F}-\mathrm{FDG}$ uptake of the primary lesions was positively correlated with that of the metastatic LNs in the thoracic field. Semiquantitative investigation using an SUVmax cutoff of 3.3 further improved the diagnostic accuracy, with higher specificity and PPVs.
The presurgical staging of primary esophageal cancer defines the therapeutic options. In particular, it is important to distinguish between patients with locoregional and systemic disease, to avoid unnecessary curative therapeutic options (3). There are multiple investigations regarding the clinical utility of ${ }^{18}$ F-FDG PET for such presurgical staging. In general, the investigators concluded that ${ }^{18} \mathrm{~F}-\mathrm{FDG}$ PET/CT had a limited role in the identification of early regional LN metastasis but was highly useful to detect remote LN involvement and metastases to the remote organs $(4,5)$. The results of the present study confirmed the low sensitivity of the visual inspection of ${ }^{18} \mathrm{~F}-\mathrm{FDG}$ PET/CT images for both regional and remote $\mathrm{LN}$ metastases.

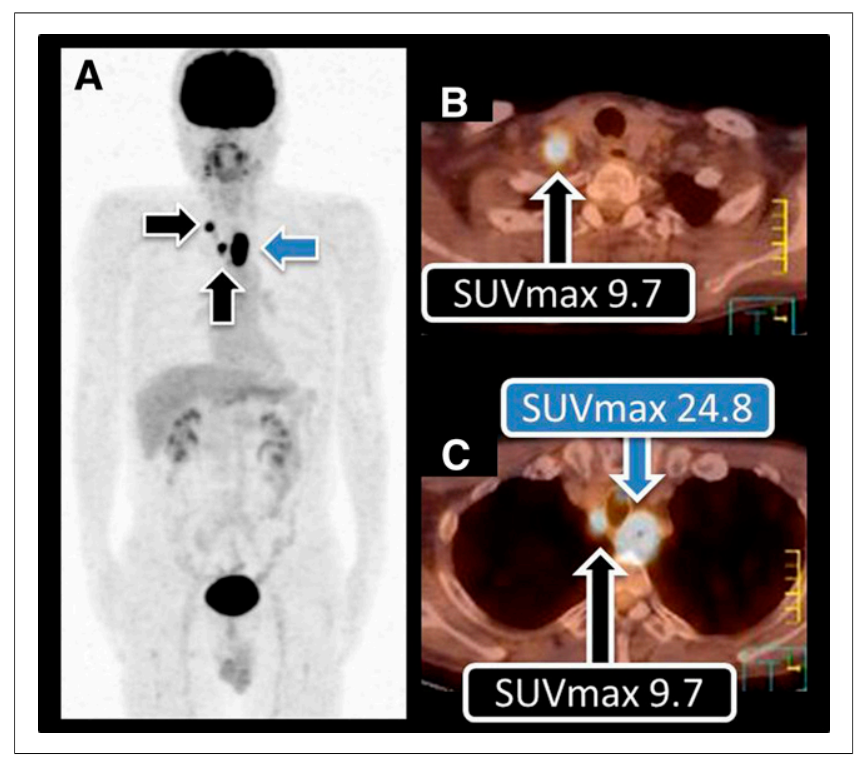

FIGURE 2. Representative ${ }^{18} \mathrm{~F}-\mathrm{FDG}$ PET images of 52-y-old man with SCC of upper thoracic esophagus showing high ${ }^{18} \mathrm{~F}-\mathrm{FDG}$ avidity in primary lesion. (A) Whole-body maximum-intensity-projection image demonstrating high ${ }^{18} \mathrm{~F}-\mathrm{FDG}$ avidity in primary lesion (blue arrow; SUVmax, 24.8) and LN uptake in the cervical and thoracic regions (black arrows). (B) Axial image of cervical region showing abnormal ${ }^{18} \mathrm{~F}-\mathrm{FDG}$ uptake in metastatic LN (SUVmax, 9.7). (C) Axial image of upper thoracic region showing abnormal ${ }^{18} \mathrm{~F}-\mathrm{FDG}$ uptake in metastatic LN (SUVmax, 9.7). Pathologic analyses disclosed T3, $\mathrm{LN}$ metastasis of cervical and thoracic regions, which were concordant with ${ }^{18} \mathrm{~F}-\mathrm{FDG} \mathrm{PET} / \mathrm{CT}$ findings. 


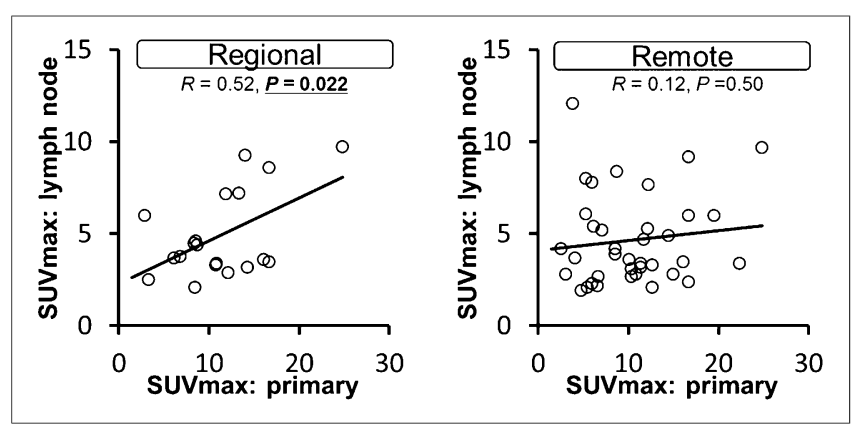

FIGURE 3. Representative SUVmax of regional and remote LN metastases was plotted against SUVmax of primary lesions. Representative value of LN SUVmax was defined as highest SUVmax of metastatic LNs in each region (cervical, thoracic, and abdominal).

The low sensitivity of PET for locoregional nodal involvement at initial staging may be related to the presence of microscopic spread, high ${ }^{18}$ F-FDG uptake in the adjacent primary tumor, or hypermetabolic activity associated with a chronic inflammatory process - all of which often coexist with the microscopic metastasis. Hybrid PET/CT imaging can overcome some of these limitations by improving the characterization of ${ }^{18} \mathrm{~F}$-FDG activity in the vicinity of highly tracer-avid primary tumors (14), but it still provides only low signals due to microscopic spread of the metastatic malignant cells. In particular, esophageal cancer with low glucose transporter 1 (Glut-1) expression accumulates less ${ }^{18} \mathrm{~F}-\mathrm{FDG}$, resulting in a low signal-to-background ratio for detecting metastatic legions. As suggested by pathologic analyses, Glut-1 expression of the metastatic LNs positively correlated with that of the primary lesions, possibly affecting the sensitivity of the PET/CT scan to detect LN metastases (15). In the present study, we reexamined the diagnostic accuracy of PET/CT to identify LN metastases in primary esophageal cancer with the correlation of the ${ }^{18} \mathrm{~F}-\mathrm{FDG}$ avidity of the primary lesions. To our knowledge, this is the first clinical investigation regarding the association of ${ }^{18} \mathrm{~F}-\mathrm{FDG}$ avidities between primary esophageal SCC and its LN metastases.

Here, the visual inspection of ${ }^{18} \mathrm{~F}-\mathrm{FDG}$ PET/CT images failed to identify the primary lesion for 21 of 73 patients with $\mathrm{T} 1$ tumors, but it succeeded in detecting all of the primary tumors more advanced than T1 stage. ${ }^{18}$ F-FDG avidity of the primary lesion was associated with tumor length, depth of invasion, macroscopic type, nodal metastasis, vascular invasion, and Glut-1 expression (16-18). Overlaps of ${ }^{18} \mathrm{~F}-\mathrm{FDG}$ avidity among T1, T2, T3, and T4 tumors probably indicated a range of Glut-1 expression (15), which in turn indicated variation in the aggressiveness of the primary lesion (19).

The detection of LN metastases is another factor affecting the clinical staging of esophageal cancer. We found that ${ }^{18}$ F-FDG PET/CT, compared with the diagnosis based on histopathologic analyses, tended to underestimate lymph nodal involvement. The underestimation was particularly clear in patients with low ${ }^{18} \mathrm{~F}$-FDG avidity in the primary lesions (SUVmax < 5). However, the ${ }^{18}$ F-FDG PET/CT images of primary lesions with higher ${ }^{18} \mathrm{~F}-\mathrm{FDG}$ avidity (SUVmax $\geq 5$ ) showed relatively better diagnostic accuracy, with a higher sensitivity for LN metastases. Previously, Mamede et al. reported a similar trend in a mixed population mostly consisting of esophageal adenocarcinoma. They reported that a pretherapeutic SUVmax greater than 12.7 complemented the visual analysis by raising the sensitivity to predict nodal metastases from $30.8 \%$ to $69.2 \%$ (20). The results of the present study were consistent with the previous investigation, although our study population included only patients with esophageal SCC. One of the explanations for the better diagnostic accuracy of LN metastasis in the tumors with higher ${ }^{18} \mathrm{~F}-\mathrm{FDG}$ avidity was their higher metabolic potential to metastasize, which affects prevalence of LN involvement (Table 3) (21). In addition, metabolically active primary lesions probably resulted in an improved sensitivity of visual inspection as an outcome of increased ${ }^{18}$ F-FDG avidity in the metastatic LNs. Theoretically, metabolic activity of the primary lesion parallels with that of the metastatic LNs, because the nodes are filled with the same type of cells as the primary. In the present study, we found no significant difference in diagnostic accuracy between medium and high ${ }^{18}$ F-FDG avidity in the primary lesion, possibly suggesting a nonlinear relationship between primary metabolic activity and the diagnostic accuracy of visual inspection of PET/CT.

Positive correlations of ${ }^{18} \mathrm{~F}$-FDG avidity between primary and regional metastatic lesions were reported in NSCLC $(7,22,23)$ and in advanced gastric cancer (24). In NSCLC, ${ }^{18}$ F-FDG uptake within the primary lesion indicated aggressiveness of the tumor, representing intratumoral lymphatic

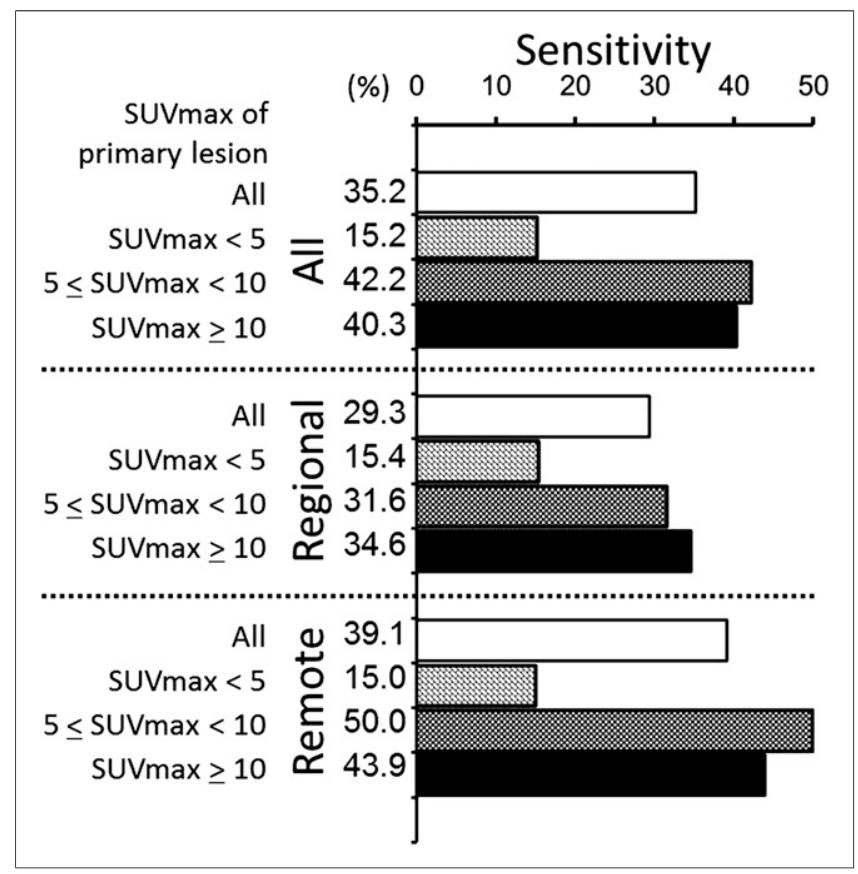

FIGURE 4. Comparison of sensitivities with visual analysis among different ${ }^{18} \mathrm{~F}-\mathrm{FDG}$ avidity in primary lesions. 

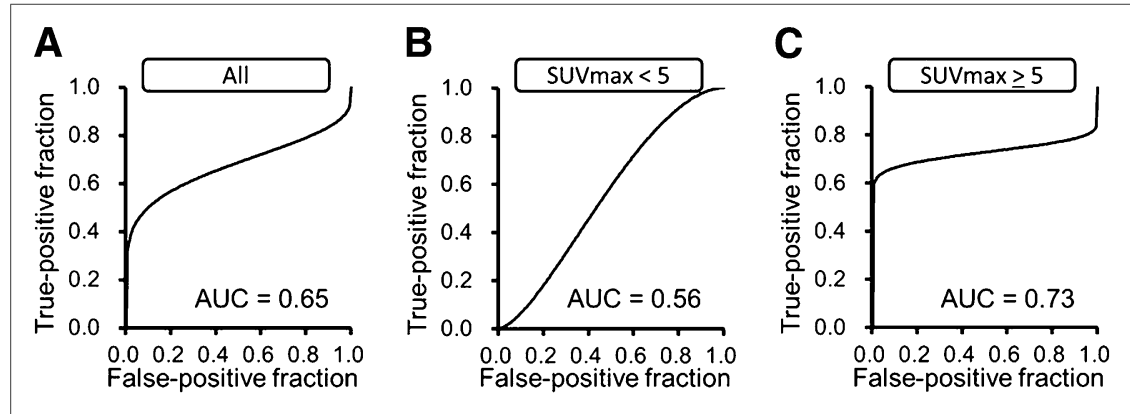

FIGURE 5. Results of ROC analyses: all patients (A), patients with low ${ }^{18} \mathrm{~F}-\mathrm{FDG}$ avidity in primary lesions (SUVmax < 5) (B), and patients with mid to high ${ }^{18} \mathrm{~F}-\mathrm{FDG}$ avidity in primary lesions (SUVmax $\geq 5$ ) (C). vessel invasion and $\mathrm{LN}$ involvement $(6,7)$. The metabolic activity of the primary tumor and tumor size are important variables in ${ }^{18} \mathrm{~F}$-FDG PET interpretation for NSCLC, because they affect the likelihood of malignant involvement in nodes (8). A similar relationship was reported in advanced gastric cancer, suggesting the possibility of falsenegative results with ${ }^{18} \mathrm{~F}$-FDG PET in primary advanced gastric cancers exhibiting low uptake of ${ }^{18}$ F-FDG (24). The results of the present study are in line with these previous investigations, and they suggest the importance of assessing the ${ }^{18} \mathrm{~F}-\mathrm{FDG}$ avidity of the primary lesions when using PET/CT for the presurgical staging of thoracic esophageal cancer. The present results did not clarify the reason for the lack of correlation between the SUVmax of the primary lesions and that of remote LNs, but the lack of correlation may suggest a difference in aggressiveness from primary lesions.

The results of our ROC analysis demonstrated that the best-cutoff value for malignant regional metastasis is the SUVmax of 3.3. This value is higher than the previously reported mean SUVmax of metastatic LNs of esophageal SCC (25). We suspected that the higher prevalence of reactive inflammatory LNs in the thoracic field elevated the optimal cutoff criterion to separate the malignant versus inflammatory pathologies. In the present study, we observed a significant overlap of SUVmax between the inflammatory and metastatic LNs, making it technically difficult to distinguish using a single cutoff value. Separating the 2 processes using a single cutoff criterion is not feasible in cases with low ${ }^{18} \mathrm{~F}-\mathrm{FDG}$ uptake of the primary cancer, because metastatic nodes probably show low ${ }^{18} \mathrm{~F}$-FDG uptake. In contrast, if a primary lesion is highly ${ }^{18} \mathrm{~F}-\mathrm{FDG}-\mathrm{avid}$, using the cutoff criterion may reasonably identify the regional metastasis.

To determine the therapeutic options, it is important to distinguish between patients with locoregional and systemic disease, because of the limited availability of curative therapeutic options for patients with distant LN metastasis or organ metastasis (3). Although there are multiple investigations debating the role of ${ }^{18} \mathrm{~F}-\mathrm{FDG}$ PET/CT for presurgical treatment planning (26), our study draws attention to the finding that primary lesions with low ${ }^{18} \mathrm{~F}$-FDG uptake have high false-negative rates for regional lymph nodal metastases, limiting the clinical utility of this technique for presurgical staging. Specifically, regional nodes could not be adequately assessed with the ${ }^{18} \mathrm{~F}-\mathrm{FDG}$ PET/CT scan if the primary tumor SUVmax was less than 5.0, because the diagnostic accuracy for identifying metastatic regional nodes is suboptimal. However, the noninvasive nature of this technique supports its clinical utility for presurgical staging, particularly with esophageal cancer with medium to high ${ }^{18} \mathrm{~F}-\mathrm{FDG}$ avidity in the primary lesions.

The present study had methodologic limitations. The pathologic analyses did not measure the size of the metastatic LNs or the diameter of the cancerous area in the LNs. Because volume information is known to improve diagnostic accuracy in LN staging (27), further investigations should examine the relation between the size of $\mathrm{LNs}$ and the ${ }^{18} \mathrm{~F}$ FDG uptake of the primary lesions.

\section{CONCLUSION}

This study showed that the avidity of the primary esophageal SCCs affected the detectability of lymph nodal metastases. If primary lesions of esophageal SCC present with a low ${ }^{18} \mathrm{~F}-\mathrm{FDG}$ uptake, PET/CT may have a limited role for initial staging because of a low sensitivity to detecting LN metastases.

\section{DISCLOSURE}

The costs of publication of this article were defrayed in part by the payment of page charges. Therefore, and solely to indicate this fact, this article is hereby marked "advertisement" in accordance with 18 USC section 1734. No potential conflict of interest relevant to this article was reported.

TABLE 5

Diagnostic Value of LN Metastasis Using Best-Cutoff Criteria in Mid to High Avidity in Primary Lesions

\begin{tabular}{lccc}
\hline \multicolumn{1}{c}{ Parameter } & $\begin{array}{c}\text { All } \\
(n=73)\end{array}$ & $\begin{array}{c}\text { Regional } \\
(n=26)\end{array}$ & $\begin{array}{c}\text { Remote } \\
(n=47)\end{array}$ \\
\hline Sensitivity (\%) & 66.1 & 70.0 & 64.1 \\
Specificity (\%) & 85.7 & 66.7 & 100.0 \\
Positive predictive value (\%) & 95.1 & 87.5 & 100.0 \\
$\begin{array}{l}\text { Negative predictive value } \\
\text { (\%) }\end{array}$ & 37.5 & 40.0 & 36.4 \\
& & & \\
\hline
\end{tabular}

Best-cutoff criteria (SUVmax, 3.3) was determined from all nodes and then applied to both regional and remote groups. 


\section{ACKNOWLEDGMENT}

We thank Masahiro Fujita for pathologic diagnosis.

\section{REFERENCES}

1. Parkin DM, Bray F, Ferlay J, et al. Global cancer statistics, 2002. CA Cancer J Clin. 2005;55:74-108.

2. Hulscher JB, van Sandick JW, de Boer AG, et al. Extended transthoracic resection compared with limited transhiatal resection for adenocarcinoma of the esophagus. N Engl J Med. 2002;347:1662-1669.

3. Wieder HA, Krause BJ, Herrmann K. PET and PET-CT in esophageal and gastric cancer. Methods Mol Biol. 2011;727:59-76.

4. Kato H, Miyazaki T, Nakajima M, et al. The incremental effect of positron emission tomography on diagnostic accuracy in the initial staging of esophageal carcinoma. Cancer. 2005;103:148-156.

5. Bruzzi JF, Truong MT, Marom EM, et al. Incidental findings on integrated PET/ CT that do not accumulate ${ }^{18}$ F-FDG. AJR. 2006;187:1116-1123.

6. Higashi K, Ueda Y, Ayabe K, et al. FDG PET in the evaluation of the aggressiveness of pulmonary adenocarcinoma: correlation with histopathological features. Nucl Med Commun. 2000;21:707-714.

7. Higashi $\mathrm{K}$, Ito $\mathrm{K}$, Hiramatsu $\mathrm{Y}$, et al. ${ }^{18} \mathrm{~F}-\mathrm{FDG}$ uptake by primary tumor as a predictor of intratumoral lymphatic vessel invasion and lymph node involvement in non-small cell lung cancer: analysis of a multicenter study. J Nucl Med. 2005;46:267-273.

8. Vesselle H, Turcotte E, Wiens L, et al. Application of a neural network to improve nodal staging accuracy with ${ }^{18} \mathrm{~F}-\mathrm{FDG}$ PET in non-small cell lung cancer. J Nucl Med. 2003;44:1918-1926.

9. Rice TW, Blackstone EH, Rusch VW. 7th ed. of the AJCC Cancer Staging Manual: esophagus and esophagogastric junction. Ann Surg Oncol. 2010;17: 1721-1724.

10. Tachibana M, Kinugasa S, Hirahara N, et al. Lymph node classification of esophageal squamous cell carcinoma and adenocarcinoma. Eur J Cardiothorac Surg. 2008;34:427-431.

11. Lamare F, Turzo A, Bizais Y, et al. Validation of a Monte Carlo simulation of the Philips Allegro/GEMINI PET systems using GATE. Phys Med Biol. 2006;51:943-962.

12. Browne J, de Pierro AB. A row-action alternative to the EM algorithm for maximizing likelihood in emission tomography. IEEE Trans Med Imaging. 1996; 15:687-699.

13. Yoon YC, Lee KS, Shim YM, et al. Metastasis to regional lymph nodes in patients with esophageal squamous cell carcinoma: CT versus FDG PET for presurgical detection prospective study. Radiology. 2003;227:764-770.
14. Bar-Shalom R, Guralnik L, Tsalic M, et al. The additional value of PET/CT over PET in FDG imaging of oesophageal cancer. Eur J Nucl Med Mol Imaging. 2005;32:918-924.

15. Hiyoshi Y, Watanabe M, Imamura Y, et al. The relationship between the glucose transporter type 1 expression and F-fluorodeoxyglucose uptake in esophageal squamous cell carcinoma. Oncology. 2009;76:286-292.

16. Nakajo M, Tani A, Kajiya Y, et al. Clinical significance of primary lesion FDG uptake for choice between oesophagectomy and endoscopic submucosal dissection for resectable oesophageal squamous cell carcinomas. Eur Radiol. 2011;21:2396-2407.

17. Miyata H, Doki Y, Yasuda $\mathrm{T}$, et al. Evaluation of clinical significance of ${ }^{18} \mathrm{~F}$ fluorodeoxyglucose positron emission tomography in superficial squamous cell carcinomas of the thoracic esophagus. Dis Esophagus. 2008;21:144-150.

18. Himeno S, Yasuda S, Shimada H, et al. Evaluation of esophageal cancer by positron emission tomography. Jpn J Clin Oncol. 2002;32:340-346.

19. Kato H, Takita J, Miyazaki T, et al. Glut-1 glucose transporter expression in esophageal squamous cell carcinoma is associated with tumor aggressiveness. Anticancer Res. 2002;22:2635-2639.

20. Mamede M, Abreu ELP, Oliva MR, et al. FDG-PET/CT tumor segmentationderived indices of metabolic activity to assess response to neoadjuvant therapy and progression-free survival in esophageal cancer: correlation with histopathology results. Am J Clin Oncol. 2007;30:377-388.

21. Gerbaudo VH, Britz-Cunningham S, Sugarbaker DJ, et al. Metabolic significance of the pattern, intensity and kinetics of ${ }^{18} \mathrm{~F}-\mathrm{FDG}$ uptake in malignant pleural mesothelioma. Thorax. 2003;58:1077-1082.

22. Li M, Liu N, Hu M, et al. Relationship between primary tumor fluorodeoxyglucose uptake and nodal or distant metastases at presentation in T1 stage non-small cell lung cancer. Lung Cancer. 2009;63:383-386.

23. Li M, Sun Y, Liu Y, et al. Relationship between primary lesion FDG uptake and clinical stage at PET-CT for non-small cell lung cancer patients: an observation. Lung Cancer. 2010;68:394-397.

24. Kim SK, Kang KW, Lee JS, et al. Assessment of lymph node metastases using ${ }^{18}$ F-FDG PET in patients with advanced gastric cancer. Eur J Nucl Med Mol Imaging. 2006;33:148-155.

25. Okada M, Murakami T, Kumano S, et al. Integrated FDG-PET/CT compared with intravenous contrast-enhanced $\mathrm{CT}$ for evaluation of metastatic regional lymph nodes in patients with resectable early stage esophageal cancer. Ann Nucl Med. 2009;23:73-80.

26. Wong WL, Chambers RJ. Role of PET/PET CT in the staging and restaging of thoracic oesophageal cancer and gastro-oesophageal cancer: a literature review. Abdom Imaging. 2008;33:183-190.

27. Roedl JB, Blake MA, Holalkere NS, et al. Lymph node staging in esophageal adenocarcinoma with PET-CT based on a visual analysis and based on metabolic parameters. Abdom Imaging. 2009;34:610-617. 